\title{
Cavity enhanced image recording for holographic data storage
}

\author{
Bo E. Miller*, Yuzuru Takashima \\ University of Arizona: College of Optical Sciences, 1630 E. University Blvd., Tucson, AZ USA \\ 85721-0094
}

\begin{abstract}
Previously, we proposed and experimentally demonstrated that optical cavities can be employed in recording and readout of plane wave holograms to improve data rates in Holographic Data Storage Systems (HDSS). However, there were some concerns about whether these techniques would be applicable to page based HDSS where signal beams are image bearing and have multiple wave vectors. We have consequently demonstrated cavity enhanced writing of image bearing holograms in Fe:LiNbO3 with a $532 \mathrm{~nm}$ wavelength, CW, single mode, DPSS, Nd:YAG, laser with a cavity on the reference arm. The diffraction efficiency was monitored by pseudo-phase-conjugate readout during the recording process.

Additionally, standing wave cavity recording was described as inappropriate to HDSS due to introducing additionally gratings to the recording process. The balancing of these grating strengths is analyzed relative to a trade-off in dynamic range consumption vs. data rates and the elimination of the extra gratings via quarter wave plates and isotropic recording media is proposed.
\end{abstract}

Keywords: Cavity, Holography, Data Storage, Standing Wave, Quarter Wave Plate, Fe:LiNbO 3 , Pseudo-phaseconjugate

\section{INTRODUCTION}

Data storage markets have shifted with the advent of cloud storage, and media streaming. Higher internet speeds have made remote data storage a viable option and reduced the need for high capacity local data storage. This shift has caused many companies to shift to remote storage like Dropbox. Thus the challenge in data storage is now to designing data centers that remotely meet the rapidly growing consumer and industrial needs.

Data center requirements come from two kinds of data, "hot" and "cold". Hot and cold data definitions are application dependent. A familiar application would be personal computers: the memory being actively used is read from long term storage and kept on fast volatile memory called Random Access Memory (RAM). In personal computers, the volatility of RAM data is clearly hot, while data remaining in long term storage is cold. Data center storage isn't so simple. Data centers store much larger quantities of data with more diverse access needs than personal computers, so it becomes meaningful to break long term storage into hot and cold archives. In archival storage, frequently accessed hot data must be stored on faster, more expensive storage media, but cold data can be kept on slower, inexpensive media to reduce the total cost of ownership of data centers. Hot data's speed requirement has led to the use of Solid State Drives (SSD) as the fastest medium available. Conversely, cold data is infrequently accessed or modified, but must be stored for long periods of time. Infrequent access coupled with the relatively high cost of SSD establishes the need to find a cheaper alternative for cold data ${ }^{1}$.

So far, magnetic tape and Hard Disc Drives (HDD) have been the cold data standard, but alternative efforts have been made to use large Blu-ray optical disc arrays ${ }^{2}$. Cooler, more efficient running, minimal backup requirements, and an order of magnitude better longevity than leading technologies give Blu-ray arrays the lowest total cost of ownership; however, such bit based, multi-layer optical storage does not scale well in capacity and data rate. Numerical Aperture, wavelength, and inter-layer absorption limit the scalability of such arrays ${ }^{4,5}$. Additionally, in optical discs readout is limited by serial access. These capacity and data rate limitations in current optical disc based cold storage are potentially overcome in Holographic Data Storage Systems (HDSS), which will likely outperform magnetic storage as well as Bluray $^{6,7}$. Various multiplexing techniques allow HDSS to accomplish such a high data capacity. The known multiplexing techniques of angular ${ }^{6,8-10}$, shift ${ }^{11}$, speckle shift ${ }^{12-14}$, and orthogonal phase code ${ }^{15}$ multiplexing provide increased capacity through multi-dimensional storage and increased speed through the parallel process of page based storage of bits ${ }^{16}$.

*millerbe31@gmail.com;

phone: 1-(747)-224-9331

Optical Data Storage 2016, edited by Ryuichi Katayama, Thomas D. Milster, Proc. of SPIE Vol. 9959, $995903 \cdot$ - (C) 2016 SPIE · CCC code: $0277-786$ X/16/\$18 $\cdot$ doi: 10.1117/12.2237078 
Further dimensionality of storage is found in phase shift keying in the coding domain ${ }^{6,17}$, where the phase sensitive nature of holography is used to encode data rather than gray scale power modulation. With increased speed and data capacity and an anticipated cost of ownership similar to Blu-ray arrays, HDSS is a very attractive solution for cold data storage.

Multidimensional storage and parallel holographic data transfer are the most attractive aspects of HDSS. However, a high degree of multiplexing limits data transfer efficiencies due to the decrease in diffraction efficiency as the number of multiplexing increases. In addition, in HDSS data is stored in the interference pattern of two beams recorded inside a photosensitive material. The recording process wastes much of the light provided for recording because only a portion of the recording light is absorbed in the writing process and the rest of it is thrown away, and similar inefficiencies take place in readout. Additionally, diffraction efficiencies of multiplexed holograms scale as $(M \# / N)^{2}$, where M\# represents the material dynamic range, and $\mathrm{N}$ is number of holograms ${ }^{18}$. Since HDSS usually multiplexes several hundred holograms the diffraction efficiency of each hologram is very low ${ }^{19}$. Typical diffraction efficiencies are less than $0.3 \%$ for readout, so most of the readout power is wasted ${ }^{19,20}$.

Conveniently, resonant optical cavities can be used to recycle the otherwise wasted energy ${ }^{21}$. This recycling can be viewed in several ways: as a means reducing the total energy cost of the system; as a means of increasing write and read data transfer rates through a reduction in exposure time; and as an easing of material sensitivity and dynamic range requirements. While it is possible to increase data transfer rates by designing more sensitive, higher dynamic range materials or increasing the power of the beams, both options have their limits which are independent from the use of cavities. The beam power cannot be increased indefinitely, and cost effective, high sensitivity, high dynamic range materials come with trade-offs related to increased shrinkage ${ }^{22,23}$. With these design limitations in mind, cavity techniques to enhance the performance of HDSS become an attractive improvement because of their minimum modification to conventional HDSS. The appeal of cavity enhancement techniques is further improved by its compatibility with the compact monocular system design ${ }^{24}$ and the improved bit error rates of pseudo-phase conjugate readout and Gaussian apodization ${ }^{16,25}$.

Previously, we developed the theory for cavity enhanced writing of blank pages and experimentally demonstrated enhanced recording speeds ${ }^{26}$. However, to address concerns about applying these techniques to page based storage we have experimentally demonstrated cavity enhanced writing of an image bearing hologram through the use of an image bearing signal arm and cavity enhanced Gaussian reference beam. The presence of extraneous gratings in cavity enhanced recording is also source of concern as they consume additional dynamic range, and we will discuss how to mitigate dynamic range loss via reference and signal power balancing. We will also discuss the use of quarter wave plates to remove the extraneous gratings entirely. Sec. 2.1 describes the experimental procedure for evaluating the cavity enhancement of the image recording, and Sec. 2.2 discusses the results of the experiment. In Sec. 3 the recording of extraneous holograms is analyzed to mitigate dynamic range consumption. Sec. 4 describes the use of quarter wave plates and isotropic media to completely remove extraneous gratings.

\section{CAVITY ENHANCED IMAGE RECORDING}

\subsection{Imaging hologram experimental procedure}

We record with a cavity in the reference arm and readout by a pseudo phase conjugate method similar to Cao et. all ${ }^{27}$. The reverse propagating beam in the standing wave cavity is used to read out the hologram while recording it.

Fig. 1 shows the experimental setup. In lieu of a data encoded bitmap pattern, we used a Newport USAF-1951 RES-1 resolution test target as the object. The object was placed at the front focal plane of a Rolyn Optics $80.3020,53 \mathrm{~mm}$ focal length, microscope objective lens, and the rear focus of the lens overlapped with the reference beam inside of the crystal. The object was illuminated by a $\sim 5 \mathrm{~mm}$ diameter Gaussian beam. Diffraction efficiency was monitored via the phase conjugate diffraction from the hologram being recorded, which was sampled with a bare microscope cover glass inserted between the object and Fourier Transform (FT) lens. The sampled diffraction was then optically chopped and lock-in amplified (EG\&G Princeton Applied Research, Model 5210) by a photodiode (Thorlabs DET36A). At the end of each recording the normal diffraction efficiency was measured without the cavity, and these measurements were used to convert the data from voltages to actual diffraction efficiencies. The reconstructed image was obtained by placing a Thorlabs DCC1445M CMOS camera at the focal plane of the FT lens during reconstruction, and the object was recorded 
by taking a picture of the signal beam at a large distance from the focal plane of the FT lens. The reference cavity was also stabilized by a proportional gain feedback loop tied to the PZT mirror and intra-cavity power monitor.

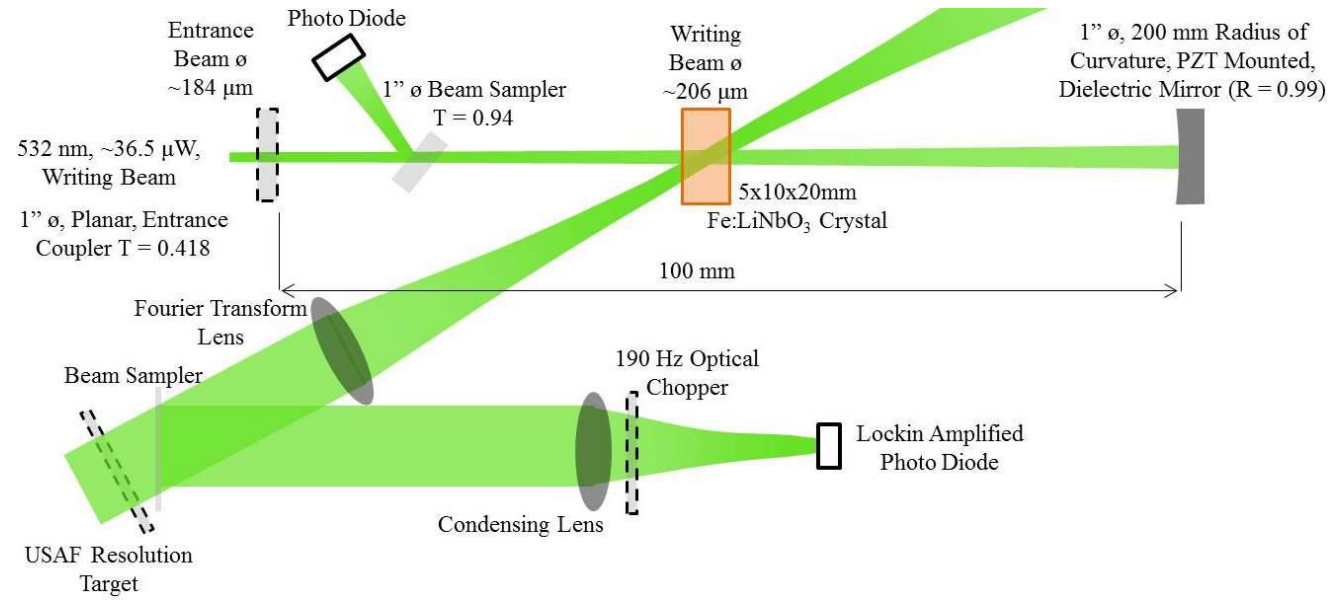

Fig. 1. Diagram of the experimental setup for cavity image recording with an enhanced reference beam.

\subsection{Imaging hologram results}

To convert the voltage data from the lock-in amplifier to actual diffraction efficiencies it is scaled according to Eq. 1:

$$
\eta_{1}(t)=\frac{1-b}{2}\left(1-\sqrt{1-\frac{4 V(t)}{P_{r e f} R_{2}(1-b)^{2} \alpha}}\right) .
$$

Here, $\eta_{1}(t)$ is the diffraction efficiency, $\mathrm{b}$ is the absorption of the crystal, $V(t)$ is the voltage data, $P_{\text {ref }}$ is the input reference beam power, $R_{2}$ is the power reflectance of the spherical mirror, and $\alpha$ is a scaling factor determined for each data set according to Eq. 2 :

$$
\alpha=\frac{V_{\max }}{P_{\text {ref }} R_{2} \eta_{\max }\left(1-b-\eta_{\max }\right)} .
$$

Here $V_{\max }$ is the maximum voltage recorded from the lock-in amplifier for the trial, and $\eta_{\max }$ is the diffraction efficiency recorded at the end of each trial. This data is fit with Eq. 3 to find the time constant $\tau$ with which the diffraction efficiency grows:

$$
\eta_{1}(t)=\sin ^{2}\left(A\left(1-e^{-\frac{t}{\tau}}\right)\right)
$$

The constant $A$ is a scaling factor related to the available dynamic range, the grating thickness, recording wavelength, and Bragg angle.

Three trial pairs, consisting of a cavity enhanced trial and unenhanced trial, were carried out as described in Sec. 2.1 with the reference arm irradiance enhanced by a factor $\sim 1.48$. Taking the square root of that value, the expected write rate enhancement is 1.22 . Write rate enhancement is computed via the ratio of the time constants, $\tau$. The mean write rate enhancement of the three trials is 1.19 with a standard deviation of 0.1 , which is $\sim 98 \%$ of the expected 1.22 . Fig. 7 displays the diffraction efficiency data for the best trial pair and a histogram showing the distribution of write rate enhancements. 


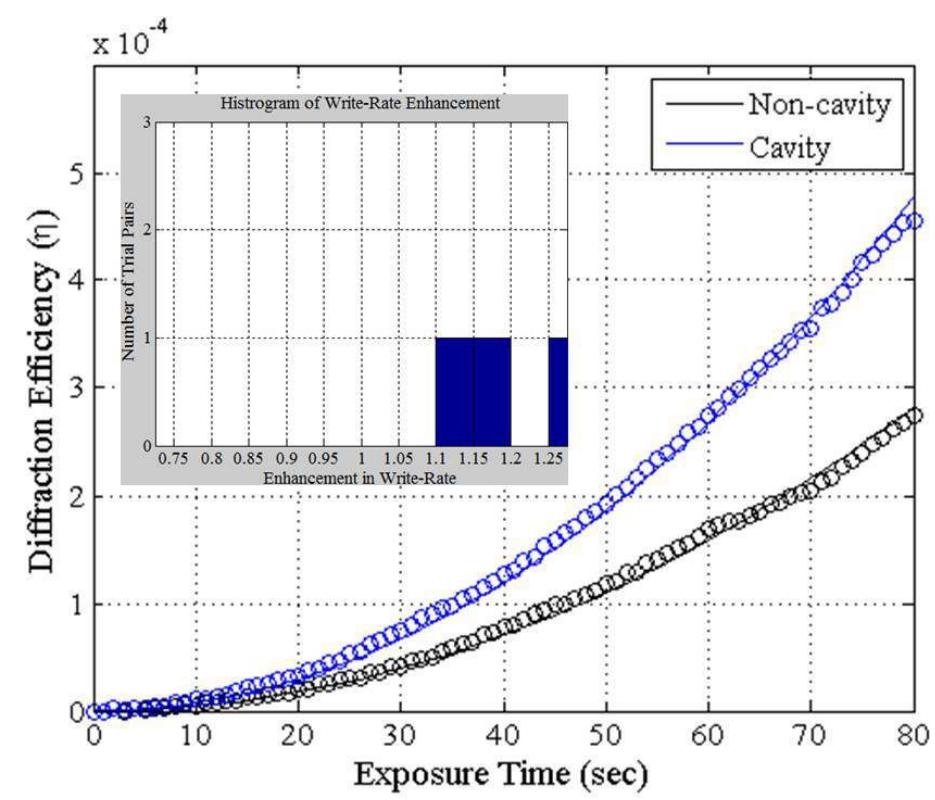

Fig. 2. Data and fitting curves for the best data set including a histogram of the enhancements. The non-cavity and cavity diffraction efficiency data have time constants of 3.34x104 sec., and $2.57 \times 104 \mathrm{sec}$, which yield a 1.30 enhancement in write data rate. The inset shows a histogram of write rate enhancements for the three trial pairs.

A representative pair of object and reconstruction images are shown in Fig. 8. The object is seen to be clearly reconstructed from the recording created with a 1.54 irradiance enhanced reference beam. We would expect a write rate enhancement of 1.24 .

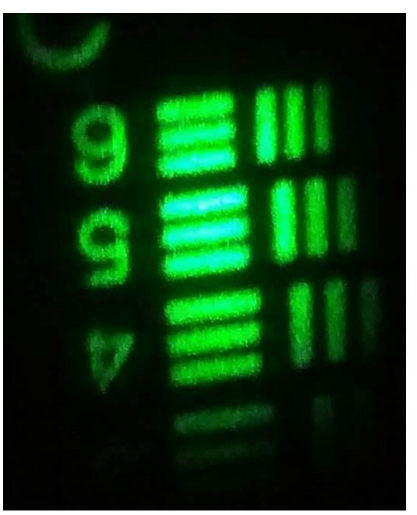

(a)

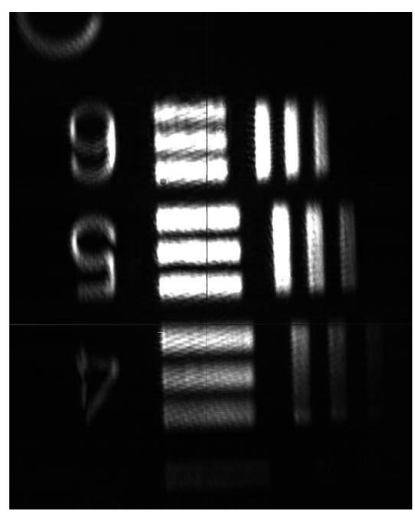

(b)

Fig. 3 (a) Object recorded: Newport USAF-1951 RES-1 group 1 elements 4 through 6. Maximum spatial frequency shown is $3.56 \mathrm{lp} / \mathrm{mm}$. (b) Reconstruction recorded with a 1.54 enhanced reference arm, anticipated write rate enhancement is 1.24.

\section{DYNAMIC RANGE CONSUMPTION OF EXTRANEOUS HOLOGRAMS}

Using a standing wave cavity to enhance the reference beam adds two additional gratings to the plane wave recording geometry by adding a counter propagating reference beam. An extra plane wave grating corresponds to extra holograms in image recording scenarios. According to Fig. 4 the two gratings are a signal bearing reflection type hologram $\vec{K}_{r e f l}$ and a distributed Bragg grating, $\vec{K}_{\text {stand }}$, containing only information about the reference beam. One could get rid of the 
standing wave by using a traveling wave resonator or quarter wave plates as in Sec. 4, but this would also get rid of the signal bearing reflection hologram.

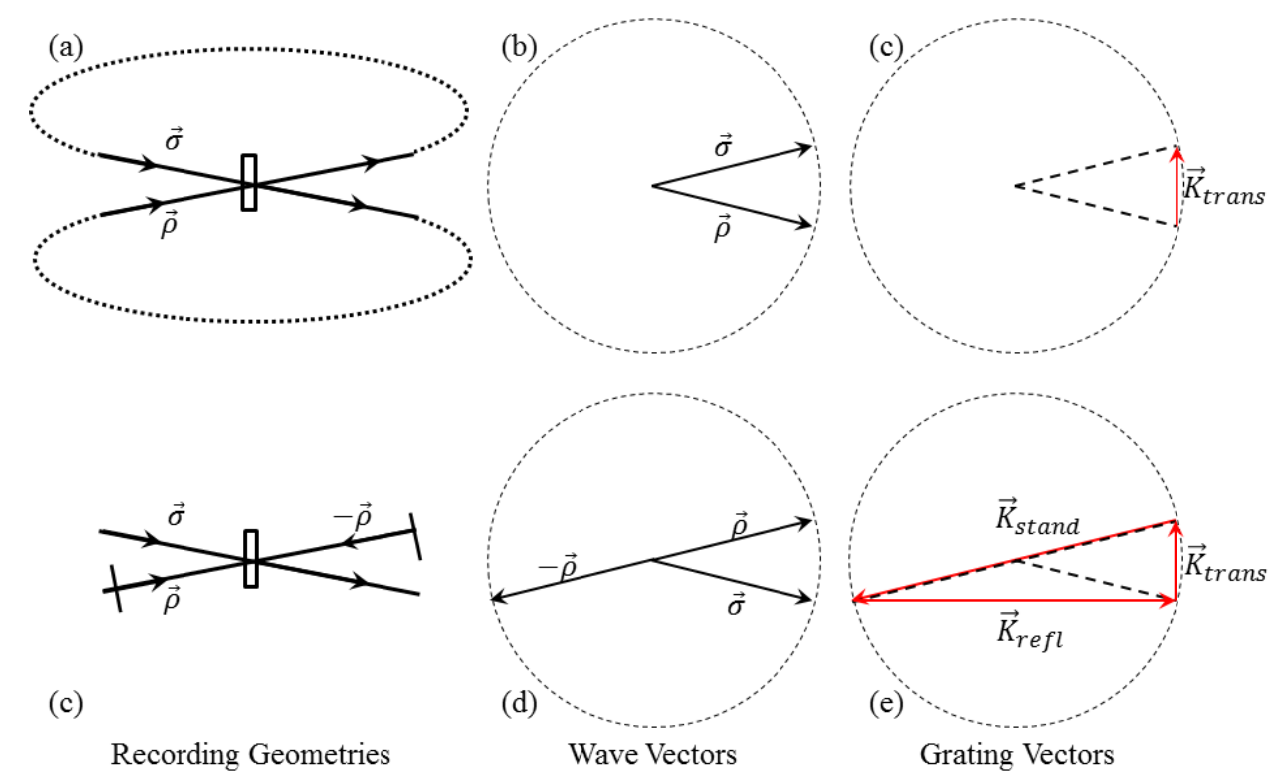

Fig. 4. Wave and grating vectors for recording geometries. (a) Recording beam geometry for normal writing as well as single and double traveling wave cavity writing. Dotted lines indicate the recirculation path of beams in traveling wave cavities. (b) Recording beam wave vectors for 4a. (c) Grating vector for 4a. (d) Recording beam geometry for single standing wave cavity writing. (e) Recording beam wave vectors for $4 \mathrm{~d}$. (c) Grating vectors for $4 \mathrm{~d}$.

Since the traveling wave cavity creates two signal bearing holograms instead of one, we can expect an additional increase in the recording speed at the cost of dynamic range consumed by the standing wave hologram. This loss of dynamic range may not be desirable, but the further increase in write rate may make it worth the loss.

It should be noted that the effect these additional gratings was not observed in our experiments due to the nature of the Fe: $\mathrm{LiNbO}_{3}$ crystal and the orientation of the c-axis relative to the grating vectors. Since the c-axis was aligned perpendicular to the reference beam, the extra gratings were oriented near perpendicular to the c-axis. While this arrangement would still allow the gratings to consume the dynamic range of the crystal, the electro-optic effect of the crystal did not cause refractive index changes to couple the recording waves ${ }^{28}$.

However, when the recording medium can support the extra gratings, such as a photo polymer, we have gratings produced according to the irradiance pattern, $E_{e, t o t}(\vec{r})$, of Eq. 4 :

$$
E_{e, t o t}(\vec{r})=\frac{E_{e, i n}}{1+S}\left[\begin{array}{c}
S G_{F}\left(1+r_{2}^{2}\left(1-b-\eta_{1}\right)\right)+1 \\
+2 S G_{F} \sqrt{r_{2}^{2}\left(1-b-\eta_{1}\right)} \cos (2 \vec{\rho} \cdot \vec{r}-\pi) \\
+2 \sqrt{S G_{F}} \cos ((\vec{\sigma}-\vec{\rho}) \cdot \vec{r}) \\
+2 \sqrt{S G_{F} r_{2}^{2}\left(1-b-\eta_{1}\right)} \cos ((\vec{\sigma}+\vec{\rho}) \cdot \vec{r}-\pi)
\end{array}\right] .
$$

Here $E_{e, \text { in }}$ is the total irradiance available to the two recording arms, $S$ is the ratio of the reference arm irradiance to the signal irradiance, $G_{F}$ is the irradiance enhancement factor of the cavity, and $r_{2}$ is the amplitude reflection coefficient of the spherical mirror. A similar equation exists for normal hologram recording:

$$
E_{e, t o t}=\frac{E_{e, i n}}{1+S}[(S+1)+2 \sqrt{S} \cos ((\vec{\rho}-\vec{\sigma}) \cdot \vec{r})] .
$$


Since the time evolution of a hologram is directly related to the irradiance of its interference pattern we can compute the ratio of the dynamic range consumed in signal recording to the dynamic range consumed by the unwanted terms like the constant offset and standing wave hologram. We will call this figure of merit the diffraction efficiency yield. For noncavity writing the yield looks like

$$
Y_{\text {non }}=\frac{2 \sqrt{S}}{1+S+2 \sqrt{S}} \text {. }
$$

Similarly, if we let $B=R_{2}\left(1-b-\eta_{1}\right)$, the yield of single standing wave recording is

$$
Y_{\text {cav }}=\frac{(1+\sqrt{B})}{1+\sqrt{B}+\frac{1}{2} \sqrt{G_{F} S}+\sqrt{B G_{F} S}+\frac{1}{2} B \sqrt{G_{F} S}+\frac{1}{2 \sqrt{G_{F} S}}} .
$$

Both yields max out at 0.5 , which corresponds to unit fringe visibility in non-cavity recording, but cavity writing has its maximum at smaller values of $\mathrm{S}$ due to the cavity enhancement. The analytic form of the optimum splitting ratio for cavity yield is

$$
S_{\text {max }, \text { cav }}=\frac{1}{G_{F}(1+2 \sqrt{B}+B)} \text {. }
$$

Using that optimum splitting ratio we arrive at Write Rate Enhancement (WRE) of

$$
W R E_{\text {opt }, \text { local }}=\sqrt{G_{F}}(1+\sqrt{B})
$$

when comparing cavity and non-cavity writing at the same splitting ratio. However, if we compare write rates at the respective maximum yield splitting ratios, $S_{\max , \text { cav }}$ for the cavity and $S=1$ for non-cavity, we see an optimal WRE of

$$
W R E_{\text {opt }, \text { global }}=\frac{\left(2 G_{F}(\sqrt{B}+1)^{2}\right)}{G_{F}+2 \sqrt{B} G_{F}+B G_{F}+1}
$$

As a point of reference we look at the optimal splitting ratio and WRE for our experimental setup: $G_{F} \cong 1.48, R_{2}=$ $0.99, b=0.139$, and $\eta_{1}$ is assumed small enough to ignore compared to $b$. In this case $S_{\text {max,cav }}=0.183$ gives us $W R E_{\text {opt, local }}=2.34$ and $W R E_{\text {opt,global }}=1.69$. Both of these WREs are improvements over conventional writing as well as the cavity enhancement described in Sec. 2. This increase in diffraction efficiency yield is show graphically in Fig. 5.

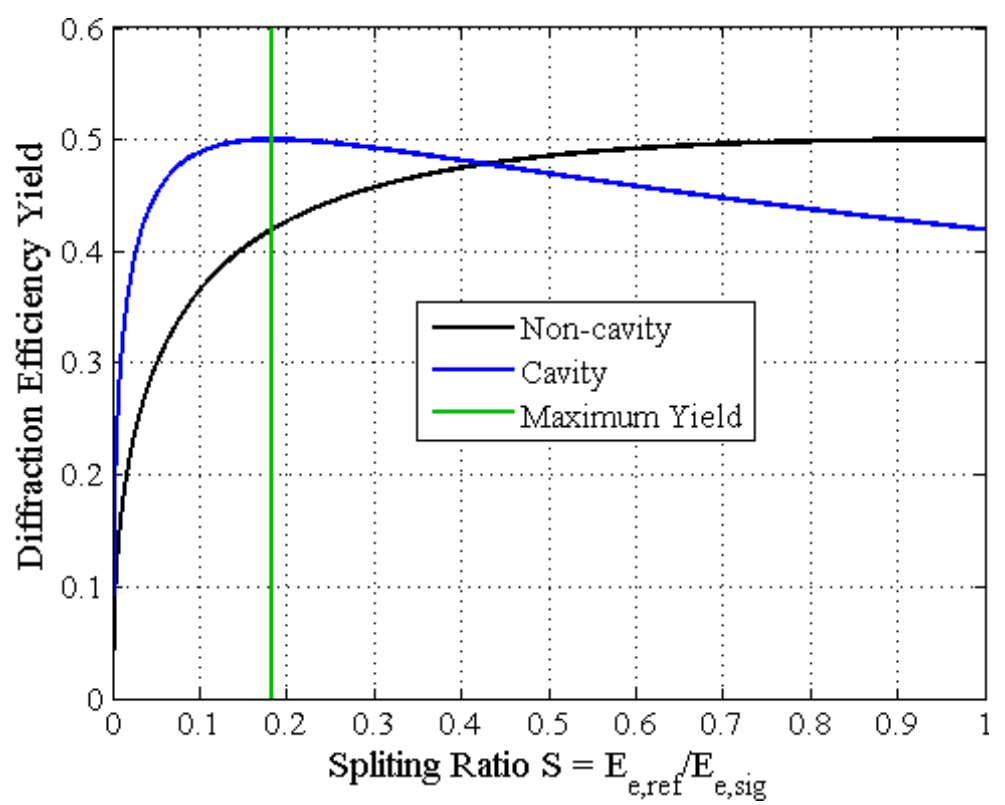

Fig. 5. Diffraction efficiency yield plotted against splitting ratio for $G_{F} \cong 1.48, R_{2}=0.99, b=0.139$, and $\eta_{1} \cong 0$. This gives $S_{\text {max }, \text { cav }}=0.183, W R E_{\text {opt }, \text { local }}=2.34$, and $W R E_{\text {opt }, \text { global }}=1.69$. 


\section{ELIMINATION OF EXTRANEOUS HOLOGRAMS}

As mentioned in Sec. 3 the extraneous holograms can be removed through the use of a traveling wave cavity, but one could also place the recording medium between two $\lambda / 4$ plates to prevent the reverse propagating reference beam from interacting with the original signal and reference beams ${ }^{29}$. This would take the form of Fig. 6 where the reference beam entering the cavity is circularly polarized. Before entering the recording medium the light is converted to a linear polarization by a $\lambda / 4$ plate. This forward propagating light in the medium has a polarization parallel to that of the signal beam. After the medium, the beam passes through a second $\lambda / 4$ plate with its fast axis rotated $45^{\circ}$ relative the direction of the linear polarization, and is converted into circular polarization. Upon reflection the beam changes handedness so that it passes back through the $\lambda / 4$ plates and medium in a polarization state orthogonal to that of the forward propagating beam. Since orthogonally polarized light cannot interfere there are no additional gratings formed by the revers propagating beam. It should be noted that this technique will only work for isotropic media because any birefringence will cause the orthogonal polarizations to deviate from each other on successive paths in the cavity which would destroy resonance.

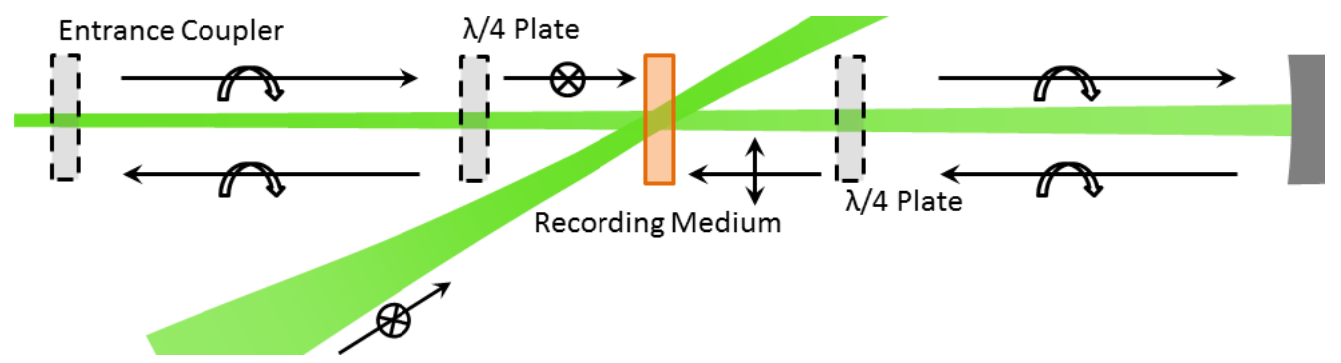

Fig. 6. $\lambda / 4$ plates are used to remove extra gratings formed in a standing wave cavity. Orthogonal polarization of the forward and reverse propagating beams prevents the formation of additional holograms due to the reverse propagating reference beam.

\section{CONCLUSIONS}

We demonstrated a 1.19x enhancement in write data rate for the recording of image holograms, thus proving that cavity enhanced recording can be applied to page passed HDSS. While our system did not display the properties of recording the extra gratings caused by a standing wave cavity, we have shown that an appropriate choice of signal and reference beam irradiance can allow for a further increase in recording speed through the use of an additional reflection type hologram. For our system, the maximum enhancement would be $1.69 \mathrm{x}$ compared to unit fringe visibility recording without a cavity. If the standing wave reflection type hologram is unacceptable, it is also possible to eliminate the extra holograms by using $\lambda / 4$ plates.

\section{REFERENCES}

[1] Clark, D., "Startups Dash Into Hot Data-Storage Sector,” Wall Str. J., 4 September 2015, $<$ http://www.wsj.com/articles/startups-dash-into-hot-data-storage-sector-1441409495> (3 October 2015 ).

[2] Verge, J., "Sony Acquires Cold Storage Blu-Ray Innovator Optical Archive," Data Cent. Knowl., 3 October 2015, <http://www.datacenterknowledge.com/archives/2015/05/27/sony-acquires-facebook-born-startuprepurposes-blu-rays-cold-storage/> (3 October 2015 ).

[3] "TCO Long-Term Archiving | DISC Archiving Systems/Blu-ray Optical Archival Storage.", <http://www.discgroup.com/technology/archive-requirements/> (25 November 2015$)$.

[4] McLeod, R. R., Daiber, A. J., McDonald, M. E., Robertson, T. L., Slagle, T., Sochava, S. L., Hesselink, L., "Microholographic multilayer optical disk data storage," Appl. Opt. 44(16), 3197 (2005).

[5] Ichimura, I., Saito, K., Yamasaki, T., Osato, K., "Proposal for a multilayer read-only-memory optical disk structure," Appl. Opt. 45(8), 1794 (2006).

[6] Anderson, K., Ayres, M., Askham, F., Sissom, B., "Holographic Data Storage: Science Fiction or Science Fact," Proc. SPIE 9201, 920102 (2014). 
[7] "Blu-ray.com - Blu-ray FAQ.", <http://www.blu-ray.com/faq/\#bluray_speed> (23 September 2015 ).

[8] Mok, F. H., "Angle-multiplexed storage of 5000 holograms in lithium niobate," Opt. Lett. 18(11), 915-917 (1993).

[9] d'Auria, L., Huignard, J. P., Slezak, C., Spitz, E., "Experimental Holographic Read-Write Memory Using 3-D Storage," Appl. Opt. 13(4), 808-818 (1974).

[10] Kinoshita, N., Muroi, T., Ishii, N., Kamijo, K., Shimidzu, N., "Control of Angular Intervals for AngleMultiplexed Holographic Memory,” Jpn. J. Appl. Phys. 48(3) (2009).

[11] Psaltis, D., Levene, M., Pu, A., Barbastathis, G., Curtis, K., "Holographic storage using shift multiplexing," Opt. Lett. 20(7), 782-784 (1995).

[12] Jeong, Y., Lee, B., "Effect of a Random Pattern through a Multimode-Fiber Bundle on Angular and Spatial Selectivity in Volume Holograms: Experiments and Theory," Appl. Opt. 41(20), 4085 (2002).

[13] Kang, Y. H., Kim, K. H., Lee, B., "Angular and Speckle Multiplexing of Photorefractive Holograms by use of Fiber Speckle Patterns,” Appl. Opt. 37(29), 6969 (1998).

[14] Kang, Y. H., Kim, K. H., Lee, B., "Volume hologram scheme using optical fiber for spatial multiplexing," Opt. Lett. 22(10), 739 (1997).

[15] Denz, C., Pauliat, G., Roosen, G., Tschudi, T., "Volume hologram multiplexing using a deterministic phase encoding method," Opt. Commun. 85(2-3), 171-176 (1991).

[16] Barbastathis, G., Psaltis, D., "Volume Holographic Multiplexing Methods," [Holographic Data Storage], D. H. J. Coufal, P. D. Psaltis, and P. G. T. Sincerbox, Eds., Springer Berlin Heidelberg, 21, 57-59 (2000).

[17] Ayres, M. R., [Signal modulation for holographic memories], University of Colorado at Boulder, Ann Arbor Michigan (2007).

[18] Mok, F. H., Burr, G. W., Psaltis, D., "System metric for holographic memory systems," Opt. Lett. 21(12), 896 (1996).

[19] Askham, F., Ayres, M. R., Urness, A. C., "High dynamic range holographic data storage media," Proc. SPIE 9587, 9587-8 (2015).

[20] Hosaka, M., Ishii, T., Tanaka, A., Koga, S., Hoshizawa, T., “1 Tbit/inch ${ }^{2}$ Recording in Angular-Multiplexing Holographic Memory with Constant Signal-to-Scatter Ratio Schedule,” Jpn. J. Appl. Phys. 52(9S2), 09LD01 (2013).

[21] Sinha, A., Barbastathis, G., "Resonant holography,” Opt. Lett. 27(6), 385-387 (2002).

[22] Takahashi, H., Naito, T., Tomita, Y., "Holographic Recording in Methacrylate Photopolymer Film Codoped with Benzyl $n$-Butyl Phthalate and Silica Nanoparticles," Jpn. J. Appl. Phys. 45(6A), 5023-5026 (2006).

[23] Ingwall, R. T., Waldman, D., "Photopolymer Systems," [Holographic Data Storage], D. H. J. Coufal, P. D. Psaltis, and P. G. T. Sincerbox, Eds., Springer Berlin Heidelberg, 195 (2000).

[24] Hoskins, A., Ihas, B., Anderson, K., Curtis, K., “Monocular Architecture,” Jpn. J. Appl. Phys. 47(7), 5912-5914 (2008).

[25] Tian, K., Barbastathis, G., "Cross talk in resonant holographic memories," J. Opt. Soc. Am. A 21(5), 751 (2004).

[26] Miller, B. E., Takashima, Y., "Enhancement of data rates by single and double cavity holographic recording," Proc. SPIE 9587, 95870G (2015).

[27] Cao, L., Zhao, Z., Gu, H., He, Q., Jin, G., "Enhancement of recording and readout for the photopolymer holographic disk system by using a conjugate structure," Proc. SPIE 6827, 68270X (2007).

[28] Yariv, A., Yeh, P., [Optical Waves in Crystals Propagation and Control of Laser Radiation], Wiley Classics Library, John Wiley \& Sons, Inc., Hoboken, New Jersey (2003).

[29] Sigel, C., Leonhardt, M. L., "System and method for providing gain and thresholding to a holographic data parallel recording and replication system incorporating independent angular address assignment," US7187481 B1 (2007). 